\title{
Design Of A Min-Sum Arborescence With Outage Costs
}

\author{
Rakesh Kawatra, Minnesota State University, Mankato, USA
}

\begin{abstract}
We present an integer programming formulation of the min-sum arborescence with node outage costs problem. The solution to the problem consists of selecting links to connect a set of terminal nodes to a root node with minimal expected annual cost, where the annual cost is the sum of annual links costs and annual outage costs. The links in the network are prone to failure and each terminal node has an associated outage cost, which is the economic cost incurred by the network user whenever that node is disabled from the central node due to failure of a link. We suggest a Lagrangian-based heuristic to get a good solution to this problem. This solution procedure also gives lower bounds to the optimal solution and is used to assess the quality of the heuristic solution. Numerical experiments taken from instances with up to 100 nodes are used to evaluate the performance of the proposed heuristic.
\end{abstract}

Keywords: Integer Programming; Heuristics; Arborescence; Networks

\section{INTRODUCTION}

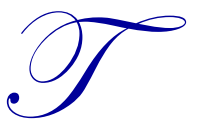

he min-sum arborescence problem consists of finding links to connect a set of geographically remote terminal nodes to a central node such that for each terminal node $\mathrm{j}$ a unique path exists from the central node to node $\mathrm{j}$. An efficient method for finding an optimal solution to this problem has been presented by Fischetti and Toth (1993). This study assumes that all terminal nodes in the network are equally valuable to the organization; but in many organizations, terminal nodes are be used for supporting an assortment of tasks like sales order entry, production planning, employee expense report processing, updating inventory records, recording quality inspection results, running decision support systems to assist managers with decision making, manufacturing process control, etc. Some of these tasks are more valuable to the organization than others. The links in a network can fail due to a variety of reasons and failure of a link can cause all the terminal nodes connected to the central node through that link to be disconnected from the network until the faulty link is repaired. The economic effect of a link failure on the organization depends upon the tasks performed on the disconnected terminal nodes. A link failure that temporarily disables a terminal node used for processing employees' expense reimbursement report may have less economic effect than a link failure which disables a computer-controlled machine (CNC) used in manufacturing. Disabling of a CNC machine may idle the machine and its operator as well as idle other machines downstream because of job starvation, and it may also result in penalties and loss of customer goodwill due to delay in shipments to the customer, whereas delay in processing employees' expense report may result in delaying payments to the employees only.

Node outage cost is the economic loss to an organization due to a terminal node's inability to communicate with the central node. It does not include the cost of repairing or replacing the link. The average yearly node outage cost is called the expected downtime cost. Campbell and Pimentel (1986) reported that, in many organizations, the expected downtime costs for some nodes can be very significant and should be taken into account while designing the topology of the network.

\section{Example}

Consider a network with four terminal nodes - 2, 3, 4, and 5 - which must be connected to a central node node 1 . The annual cost of directly linking node $\mathrm{i}$ and node $\mathrm{j}, \mathrm{C}_{\mathrm{ij}}$, as well as the outage cost associated with each 
terminal node $\mathrm{i}$ is given. We assume $\mathrm{C}_{\mathrm{ij}}=\mathrm{C}_{\mathrm{j}}, \forall \mathrm{i}, \mathrm{j}$. We assume a link failure rate of 0.10 for each link. (The values of link costs and node outage costs are given in Table 1.)

Table 1: Link And Outage Costs For The 5-Node Problem

\begin{tabular}{|c|c|c|c|c|c|c|}
\hline $\mathbf{i}$ & $\mathbf{C}_{\mathbf{i} 1}$ & $\mathbf{C}_{\mathbf{i} 2}$ & $\mathbf{C}_{\mathbf{i} 3}$ & $\mathbf{C}_{\mathbf{i} 4}$ & $\mathbf{C}_{\mathbf{i} 5}$ & Node Outage Cost \\
\hline 2 & 25 & --- & 16 & 8 & 20 & 500 \\
\hline 3 & 36 & 16 & --- & 14 & 10 & 100 \\
\hline 4 & 27 & 8 & 14 & --- & 12 & 200 \\
\hline 5 & 35 & 20 & 10 & 12 & --- & 700 \\
\hline
\end{tabular}

Using only the link costs, this network would be optimally configured as shown in Figure 1a. The total annual link cost of this configuration is 57 , but the total annual cost including the expected downtime costs is 437 . Although the annual link cost for the configuration in Figure $1 \mathrm{~b}$ is higher at 101, the total annual cost, including the expected downtime cost, is 261 , which is $40 \%$ lower than the corresponding cost for the layout in Figure 1a. Thus, node outage costs can have a significant effect on the total annual cost of the network and should be considered during the topological design of the network.
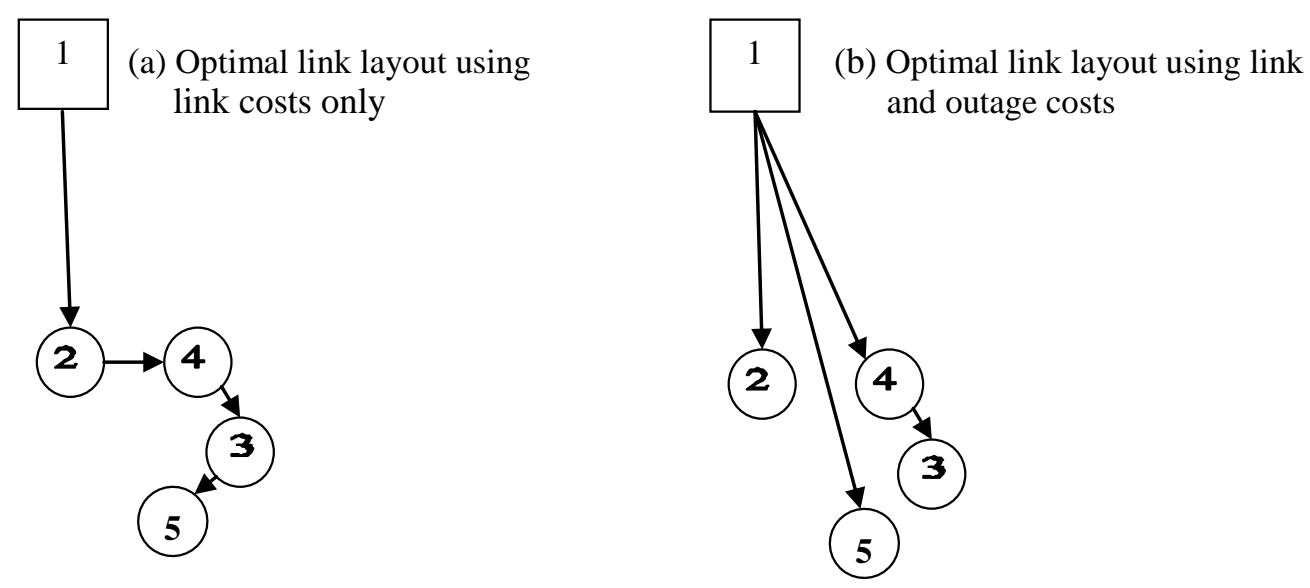

Figure 1: A 5-node Problem

The network design problem is formulated as an integer program. We propose a Lagrangian relaxation based heuristic to find a good solution to this problem. The rest of the paper is organized as follows. In Section 2, we present an integer programming formulation of the min-sum arborescence with node outage costs problem. Section 3 presents our Lagrangian relaxation heuristic for obtaining a lower bound of the optimal cost. In Section 4, we present a branch exchange heuristic, which is used after each iteration of the Lagrangian relaxation method to give good feasible solutions. In Section 5, we present the results reflecting the performance of our heuristic algorithm.

\section{PROBLEM FORMULATION}

We formulate the min-sum arborescence problem with node outage cost as an integer-programming problem. The objective is to find a solution that has minimum total annual cost consisting of links costs and the node outage costs. In the problem, the links are directed away from the central node toward the terminal nodes.

We use the following notations in the paper:

- $\quad$ S: the set of terminal nodes $2,3 \ldots \mathrm{N}$

- $\quad$ Node 1: central node

- $\quad \operatorname{link}(\mathrm{i}, \mathrm{j})$ : a directed link from node $\mathrm{i}$ to node $\mathrm{j}$

- $\quad \mathrm{C}_{\mathrm{ij}}$ : annual cost of installing a link(i,j) 
- $\quad \mathrm{O}_{\mathrm{k}}$ : node outage cost associated with terminal node $\mathrm{k}$

- f: link failure rate

- Decision Variables

$X_{i j}=\left\{\begin{array}{l}1 \quad \text { if link }(i, j) \text { is in the solution }, \\ 0 \quad \text { otherwise }\end{array}\right.$

$Y_{i j}^{k}= \begin{cases}1 & \text { if } \operatorname{link}(i, j) \text { is on the path from the central node to node } k, \\ 0 & \text { otherwise }\end{cases}$

The min-sum arborescence problem with node outage costs can be represented mathematically as the following integer programming problem:

$\mathrm{Z}=\operatorname{Minimum}\left\{\sum_{\mathrm{i}=1}^{\mathrm{N}} \sum_{\mathrm{j}=2}^{\mathrm{N}} \mathrm{C}_{\mathrm{ij}} \mathrm{X}_{\mathrm{ij}}+\sum_{\mathrm{k}=2}^{\mathrm{N}} \mathrm{O}_{\mathrm{k}} \sum_{\mathrm{i}=1}^{\mathrm{N}} \sum_{\mathrm{j}=2}^{\mathrm{N}} \mathrm{f} \mathrm{Y}_{\mathrm{ij}}^{\mathrm{k}}\right\}$

subject to

$\sum_{\mathrm{i}=1}^{\mathrm{N}} \mathrm{X}_{\mathrm{ij}}=1 \quad$ for all $\mathrm{j} \in \mathrm{S}$

$\sum_{j=2}^{N} Y_{i j}^{k}-\sum_{j=1}^{N} Y_{j i}^{k}=\left\{\begin{array}{l}+1 \text { if } i=1 \\ -1 \text { if } i=k \\ 0 \text { otherwise }\end{array} \quad\right.$ for all $i \in S \cup[1], k \in S$

$\mathrm{Y}_{\mathrm{ij}}^{\mathrm{k}} \leq \mathrm{X}_{\mathrm{ij}}$

for all $i \in S \cup[1]$, and $j, k \in S$

$\sum_{i=1}^{N} \sum_{j=2}^{N} X_{i j}=N-1$

$\mathrm{X}_{\mathrm{ij}} \in\{0,1\}$

for all $i \in S \cup[1]$, and $j \in S$

$\mathrm{Y}_{\mathrm{ij}}^{\mathrm{k}} \in\{0,1\}$

for all $\mathrm{i} \in \mathrm{S} \cup[1]$, and $\mathrm{j}, \mathrm{k} \in \mathrm{S}$

The two terms in the objective function represent the total annual cost of the links and the total expected downtime costs, respectively. The expected downtime cost for a node is given by the product of its outage cost and the failure rate of the path from the central node, which is given by the sum of individual failure rates of the links that make up the path. Constraints (3) are flow conservation constraints. Constraints (3), (4) and (5) ensure that the solution does not contain circuits.

We propose a Lagrangian relaxation-based heuristic method to solve this problem. This method also gives a lower bound that can be used to obtain a quantitative estimate of the quality of the heuristic solution. An application-oriented survey of Lagrangian relaxation may be found in Fisher (1981). Lagrangian relaxation-based heuristics have been used very successfully by Gavish (1982), Gavish (1985), Pirkul et al. (1988), Kawatra (2002), and Volgenant (1989) for finding good solutions to difficult network design problems.

\section{LAGRANGIAN RELAXATION}

For our heuristic, we first form a Lagrangian relaxation of the min-sum arborescence problem with node outage costs by multiplying each constraint (4) by a non-negative Lagrange multiplier $\mu_{\mathrm{ijt}}$ and adding the products to 
the objective function. By relaxing these constraints, the problem decomposes into two sets of sub-problems: 1) a link selection sub-problem denoted as $\operatorname{LS}(\mu)$, and 2) (N-1) routing sub-problems denoted as $\mathrm{R}_{\mathrm{k}}(\mu)-$ for $\mathrm{k}=2,3, \ldots . \mathrm{N}$. These sub-problems can be individually solved for optimal solutions. For a given set of Lagrange multipliers, the cost given by the Lagrange solution is used as a lower bound on the optimal cost of the original problem $\mathrm{Z}$. Usually, the topological solution given by $\operatorname{LS}(\mu)$ would have high total expected downtime costs resulting in high total annual cost. We use a branch exchange heuristic to iteratively reduce the total annual cost of the network. For non-negative multipliers $\mu$ the Lagrangian relaxation of the problem $\mathrm{Z}$ is:

$\mathrm{L}(\mu)=\operatorname{Minimize}\left\{\sum_{\mathrm{i}=1}^{\mathrm{N}} \sum_{\mathrm{j}=2}^{\mathrm{N}} \mathrm{X}_{\mathrm{ij}}\left(\mathrm{C}_{\mathrm{ij}}-\sum_{\mathrm{k}=2}^{\mathrm{N}} \mu_{\mathrm{ijk}}\right)+\sum_{\mathrm{k}=2}^{\mathrm{N}} \sum_{\mathrm{i}=1}^{\mathrm{N}} \sum_{\mathrm{j}=2}^{\mathrm{N}}\left(\mu_{\mathrm{ijk}}+\mathrm{O}_{\mathrm{k}} \mathrm{f}\right) \mathrm{Y}_{\mathrm{ij}}^{\mathrm{k}}\right\}$ subject to (2), (3), (5), (6) and (7).

This can be separated into

$\operatorname{LS}(\mu)=\operatorname{Minimize}\left\{\sum_{\mathrm{i}=1}^{\mathrm{N}} \sum_{\mathrm{j}=2}^{\mathrm{N}} \mathrm{X}_{\mathrm{ij}}\left(\mathrm{C}_{\mathrm{ij}}-\sum_{\mathrm{k}=2}^{\mathrm{N}} \mu_{\mathrm{ijk}}\right)\right\}$ subject to (2), (5) and (6); and,

for $\mathrm{k}=2,3, \ldots . \mathrm{N}$

$\mathrm{R}_{\mathrm{k}}(\mu)=\operatorname{Minimize}\left\{\sum_{\mathrm{i}=1}^{\mathrm{N}} \sum_{\mathrm{j}=2}^{\mathrm{N}}\left(\mu_{\mathrm{ijk}}+\mathrm{O}_{\mathrm{k}} \mathrm{f}\right) \mathrm{Y}_{\mathrm{ij}}^{\mathrm{k}}\right\}$ subject to

$\sum_{j=2}^{N} Y_{i j}^{k}-\sum_{j=1}^{N} Y_{j i}^{k}=\left\{\begin{array}{ll}+1 & \text { if } i=1 \\ -1 & \text { if } i=k \\ 0 & \text { otherwise }\end{array} \quad\right.$ for all $i \in S \cup[1]$

$\mathrm{Y}_{\mathrm{ij}}^{\mathrm{k}} \in\{0,1\} \quad$ for all $\mathrm{i} \in \mathrm{S} \cup[1]$, and $\mathrm{j} \in \mathrm{S}$

\section{Link Selection Sub-problem LS $(\mu)$}

For any given set of Lagrange multipliers $\mu$, we define modified cost $\widetilde{\mathrm{C}}_{\mathrm{ij}}=\left(\mathrm{C}_{\mathrm{ij}}-\sum_{\mathrm{t}=2}^{\mathrm{N}} \mu_{\mathrm{ijk}}\right)$. The link selection sub-problem LS $(\mu)$ can now be expressed as:

$\operatorname{Minimize}\left\{\sum_{\mathrm{i}=1 \mathrm{j}=2}^{\mathrm{N}} \sum_{\mathrm{ij}}^{\mathrm{N}} \widetilde{\mathrm{C}}_{\mathrm{ij}} \mathrm{X}_{\mathrm{ij}}\right\}$ subject to (2), (5) and (6).

LS $(\mu)$ can be solved by finding the min-sum arborescence $\hat{X}(\mu)$ rooted at node 1 , using the algorithm proposed by Fischetti and Toth (1993).

\section{Routing Sub-problem $\mathrm{R}_{\mathrm{k}}(\mu)$}

$\mathrm{R}_{\mathrm{k}}(\mu)$ is a single-commodity flow problem. In this problem, one unit of a commodity $\mathrm{k}$ is to be shipped from the central node to node $\mathrm{k}$. Since the links are uncapacitated, in the optimal solution the flow $\hat{\mathrm{Y}}_{\mathrm{k}}(\mu)$ will be along the shortest path from the central node to node $\mathrm{k}$, which can be found using the Dijkstra's algorithm given in Larson and Odoni (1981) with $\left(\mu_{\mathrm{ijk}}+\mathrm{f}^{*} \mathrm{O}_{\mathrm{k}}\right)$ as the length of the arc $(\mathrm{i}, \mathrm{j})$. While solving $\mathrm{R}_{\mathrm{k}}(\mu)$, we stop the Dijkstra's algorithm as soon as a shortest path to node $\mathrm{k}$ is found. 
The optimal value $L(\mu)$ of the complete Lagrangian sub-problem is the sum of (a) the link selection subproblem optimal value and (b) the optimal values $R_{k}(\mu)$ of the routing sub-problems for all $k \in S$. We use subgradient optimization method suggested by Held et al. (1974) to find the highest possible $L(\mu)$ which represents lower bound to the optimal value of the original problem $\mathrm{Z}$.

\section{A BRANCH EXCHANGE HEURISTIC}

We apply a branch exchange heuristic to the solution given by $\operatorname{LS}(\mu)$ after each iteration. Although the solution given by $\operatorname{LS}(\mu)$ is feasible, it is likely to have very high total annual cost due to high expected downtime costs. This branch exchange heuristic attempts to reduce the annual cost of the network while maintaining feasibility.

For this heuristic, we define the following additional notations:

$\prod_{i k}=\{(\mathrm{i}, \mathrm{j}) \mid(\mathrm{i}, \mathrm{j})$ is on the path from node $\mathrm{i}$ to node $\mathrm{k}\}$ for all $\mathrm{i}, \mathrm{k} \in \mathrm{S}$

Level $\mathrm{j}$ : number of links between the central node and node $\mathrm{j}$

$B^{2}=\left\{k \mid\right.$ Level $_{k} \geq 2$ for all $\left.k \in S\right\}$; i.e., the set of nodes that are at least two links from the central node

$\mathrm{p}_{\mathrm{j}}=\left\{\mathrm{i} \mid \mathrm{X}_{\mathrm{ij}}=1\right\}$ for all $\mathrm{j} \in \mathrm{S}$; i.e., $\mathrm{p}_{\mathrm{j}}$ is the node where the link ending at node $\mathrm{j}$

$\psi_{\mathrm{i}}=\sum\left\{\mathrm{O}_{\mathrm{k}} \mid\left(\mathrm{p}_{\mathrm{k}}, \mathrm{k}\right) \in \prod_{\mathrm{ik}}\right\}$; i.e., the sum of outage costs of the terminal nodes on the subtree rooted at node $\mathrm{i}$

Initially, $X=\widehat{X}(\mu)$. Note that since all of the values above are dependent upon the value of $X$, they must be recomputed each time $\mathrm{X}$ is modified by replacement of a link.

Step 1: For each $\mathrm{j} \in B^{2}$ and $\mathrm{i} \in \mathrm{S} \cup[1]$ compute

$\Delta_{\mathrm{ij}}=\left\{\mathrm{C}_{\mathrm{ij}}-\mathrm{C}_{\mathrm{f}_{\mathrm{j}} \mathrm{j}}+\left(\right.\right.$ Level $_{\mathrm{j}}-$ Level $\left.\left._{\mathrm{i}}+1\right) * \mathrm{f}^{*} \psi_{\mathrm{j}}\right\}$

Step 2: Compute $\left(i^{*}, j^{*}\right)=\underset{\mathrm{i}, \mathrm{j}}{\operatorname{Argmin}}\left\{\Delta_{i j}\right\}$

Step 3: If $\Delta_{i^{*}, j^{*}} \geq 0$, replace link $\left(f_{j^{*}}, j^{*}\right)$ with link $\left(i^{*}, j^{*}\right)$ and return to Step 1; else STOP.

\section{NUMERICAL RESULTS}

We investigated the effectiveness of the Lagrangian-based heuristic by solving a set of test problems with the number of nodes varying from 20 to 100 . The coordinates of the terminal nodes and the central node were obtained from the CRD data sets provided by Craig et al. (1996). For the annualized cost of installation of link(i,j), we used the Euclidean distance between points $i$ and $j$, while the outage costs associated with the terminal nodes of each of the CRD problems were drawn from a uniform distribution U[1,1000]. For each test problem, the failure rate $\mathrm{f}$ was uniform over the links and was varied from 0.02 to 0.10 . The branch exchange heuristic method and the subgradient optimization method were coded in FORTRAN and run on Intel Xeon cluster with 2,394 MHz processing speed.

The initial values of the Lagrangian multipliers were set at zero and modified using subgradient optimization method. After each iteration, an upper bound $\bar{Z}$ and a lower bound $\underline{Z}$ (the best-known heuristic solution and the best-known Lagrangian relaxation, respectively) are maintained. The upper bound $\bar{Z}$ is used in computing the subgradient stepsize. The value of the stepsize parameter $\lambda$ was initially set to 2 and halved if in 30 
successive iterations there was no improvement in the lower bound $\underline{Z}$. The search for the optimal Lagrangian multipliers was terminated if the total number of iterations exceeded 800 or if the $\underline{Z}$ improved by less than 0.1 in 30 successive iterations.

The computational results indicate that the Lagrangian-based heuristic consistently gives good solutions. The gaps between the best feasible solution value and the lower bound given by the Lagrangian heuristic are reported in Table 2 and are used to judge the quality of the heuristic solutions. Since the gap between the optimal solution and the heuristic solution can be no greater than this gap and is likely to be smaller, the results indicate that the heuristic solutions are within 20 percent of the optimal solution. The quantity I is the ratio of the total annual costs (including expected outage costs) of the two solutions, best network layout found using just the link costs and the best network layout found using both the link costs and node outage costs using suggested heuristic. A value of $\mathrm{I}=2$, for example, would indicate that ignoring the outage costs in the process of designing the communication network would result in a doubling of the total annual costs.

Table 2: Computational Results

\begin{tabular}{|l|c|c|c|}
\hline No. Of Nodes & Link Failure Rate & Gap & I \\
\hline & \multicolumn{2}{|c|}{ Central Node In The Corner } & 1.03 \\
\hline 20 & 0.02 & $0.2 \%$ & 1.11 \\
\hline 20 & 0.06 & $1.0 \%$ & 1.18 \\
\hline 20 & 0.10 & $2.3 \%$ & 1.09 \\
\hline 60 & 0.02 & $7.3 \%$ & 1.37 \\
\hline 60 & 0.06 & $12.5 \%$ & 1.63 \\
\hline 60 & 0.10 & $13.9 \%$ & 1.32 \\
\hline 100 & 0.02 & $15.0 \%$ & 1.95 \\
\hline 100 & 0.06 & $18.8 \%$ & 2.45 \\
\hline 100 & 0.10 & $19.3 \%$ & 1.01 \\
\hline & & & 1.05 \\
\hline 20 & 0.02 & $0.1 \%$ & 1.10 \\
\hline 20 & 0.06 & $0.4 \%$ & 1.12 \\
\hline 20 & 0.10 & $0.6 \%$ & 1.42 \\
\hline 60 & 0.02 & $4.7 \%$ & 1.68 \\
\hline 60 & 0.06 & $8.2 \%$ & 1.34 \\
\hline 60 & 0.10 & $9.4 \%$ & 1.97 \\
\hline 100 & 0.02 & $11.9 \%$ & 2.47 \\
\hline 100 & 0.06 & $14.1 \%$ & \\
\hline 100 & 0.10 & $13.9 \%$ & \\
\hline
\end{tabular}

Gap $=$ (heuristic solution value - lower bound $) /($ heuristic solution value $)$

\section{CONCLUSIONS}

In this paper, we presented an integer programming model of a min-sum arborescence problem with node outage costs. A Lagrangian relaxation method to find a lower bound of the optimal solution is given. The subgradient optimization method is used to find Lagrangian multipliers providing the best lower bound. A branch exchange heuristic embedded in the Lagrangian relaxation method is used to find a good feasible solution to the original problem. The best feasible solution is retained when the Lagrangian relaxation method stops. The best lower bound given by the Lagrangian relaxation method is used to estimate the quality of the branch exchange heuristic solution. Computational results show that the gap between the lower bound and the heuristic solution is within 20 percent.

\section{ACKNOWLEDGEMENT}

The author would like to thank the Minnesota Supercomputing Institute for their computational support. 


\section{AUTHOR INFORMATION}

Rakesh Kawatra is a professor of information systems at Minnesota State University, Mankato. He has a BTech. from IIT, Delhi, MBA from IIM, Bangalore, and PhD from The University of Iowa. His work has been published in European Journal of Operational Research, Computers and Operations Research, and other journals. His research focuses on topological design of communication networks. E-mail: rakesh.kawatra@mnsu.edu

\section{REFERENCES}

1. Campbell, G. F., and Pimentel, J. R. 1986. "Topological Aspects of MAP Network Design", Proceedings of the $11^{\text {th }}$ Conference on Local Computer Networks, pp. 34-43.

2. Craig, G., Krishnamoorthy, M., and Palaniswami, M. 1996. "Comparison of heuristic algorithms for the degree constrained minimum spanning tree," Metaheuristics: Theory and Applications, I.H. Osman and J.P. Kelly (eds.), Kluwer, Boston.

3. Fischetti, M. and Toth P. 1993. "An efficient algorithm for the min-sum arborescence problem on complete digraphs," ORSA Journal on Computing, vol. 5, no. 4, pp. 426-434.

4. $\quad$ Fisher, M.L. 1981. "The Lagrangian relaxation method for solving integer programming problems", Management Science, vol. 27, 1-18.

5. Gavish, B. 1982. "Topological Design of Centralized Computer Networks, Formulations and Algorithms", Networks, vol.12, pp. 355-377.

6. Gavish, B. 1985. "Augmented Lagrangian Based Algorithm for Centralized Network Design", IEEE Transaction on Communications, vol. 33, pp. 1247-1257.

7. Held, M., Wolfe, P., and Crowder, H.D. 1974. "Validation of Subgradient Optimization", Mathematical Programming, vol. 6, 62-88.

8. Kawatra, R. 2002. “A Multiperiod Degree Constrained Minimal Spanning Tree Problem”, European Journal of Operational Research, vol. 143, no. 1.

9. $\quad$ Larson, R. and Odoni, A. 1981. "Urban Operations Research", Prentice Hall, Englewood Cliffs, N. J..

10. Pirkul H., Narasimhan, S., and De, P. 1988. "Locating Concentrators for Primary and Secondary Coverage in a Computer Communications Network", IEEE Transactions on Communications, vol. 36, pp. 450-457.

11. Volgenant, A. 1989. "A Lagrangian approach to the degree-constrained minimum spanning tree problem", European Journal of Operational Research, vol. 39, pp. 325-331. 


\section{NOTES}

